\title{
Influence of tibial slope on extension and flexion gaps in total knee arthroplasty: increasing the tibial slope affects both gaps
}

\author{
Andrej Maria Nowakowski • Marcus Kamphausen • \\ Geert Pagenstert • Victor Valderrabano • \\ Magdalena Müller-Gerbl
}

Received: 9 February 2014 / Accepted: 2 May 2014 /Published online: 25 May 2014

(C) The Author(s) 2014. This article is published with open access at Springerlink.com

\begin{abstract}
Purpose Increasing the tibial slope is often performed if the flexion gap is narrower than the extension gap. The main hypothesis of this study is that increasing the tibial slope coincidentally enlarges the extension gap.

Methods Twenty formalin-fixed cadaveric knees were obtained for study. After CT in full extension and $90^{\circ}$ flexion, the data of each specimen were entered into a standardized coordinate system and virtual bone cuts were performed with incrementally increasing the posterior slope. Gaps were measured at tibiofemoral contact points in $90^{\circ}$-flexion and full extension in the medial and lateral compartment.

Results Increasing the tibial slope did significantly widen both the extension and the flexion gaps $(p<0.001)$. In extension, the opening rates, i.e. the gap increase per degree of slope increase, were equal medially and laterally $(0.5 \mathrm{~mm}$ $\pm 0.1)$ medial vs $0.6 \mathrm{~mm}( \pm 0.0)$ lateral), whereas in flexion the lateral gap did open significantly more than the medial one $(0.6 \mathrm{~mm} \pm 0.1)$ medial vs $0.9 \mathrm{~mm}( \pm 0.1)$ lateral $(p<0.001)$, resulting in a significantly greater flexion gap laterally.
\end{abstract}

A. M. Nowakowski $(\bowtie) \cdot G$. Pagenstert $\cdot$ V. Valderrabano Orthopaedic Department, University of Basel, Spitalstrasse 21, 4031 Basel, Switzerland

e-mail: andrej.nowakowski@unibas.ch

G. Pagenstert

e-mail: geert.pagenstert@usb.ch

V. Valderrabano

e-mail: victor.valderrabano@usb.ch

A. M. Nowakowski • M. Kamphausen • M. Müller-Gerbl Institute of Anatomy, University of Basel, Pestalozzistrasse 20 , 4056 Basel, Switzerland

M. Kamphausen

e-mail: kamphausen.marcus@googlemail.com

M. Müller-Gerbl

e-mail: m.mueller-gerbl@unibas.ch
Conclusions Increasing the tibial slope beyond the preoperative planning in order to widen a tight flexion gap intra-operatively is not recommended as doing so will increase the extension gap simultaneously and will make the medial and lateral flexion gaps unequal.

Keywords Total knee arthroplasty · Ligament balancing · Flexion gap · Extension gap · Gap kinematic

\section{Introduction}

In total knee arthroplasty (TKA), implant alignment in flexion and extension and correct sizing are essential for good ligament/soft-tissue tension and postoperative function [1]. Many surgeons strive to produce extension and flexion gaps that are of equal magnitude and/or symmetrical [2-5].

Krackow et al. [6] and Mihalko et al. [7] defined the tibiofemoral gap as it occurs under distraction of the adjacent soft-tissue mantle. The flexion and extension gaps are influenced by various factors, including deformities and osteolysis [4], contractures of the soft tissue mantle [8], operative approach [9], osteophytes [10,11], and eversion of the patella $[12,13]$. Even preparation of the flexion gap can influence the magnitude of the extension gap [14]. Finally, bony resection can affect capsular and ligamentous tension, and thus also the size of the gaps [15].

In a cadaveric study, Bellemans et al. [16] found that increasing the tibial slope improves knee flexion. Lombardi et al. [17] reported that in cases of a relatively tight flexion gap, equalizing flexion and extension gap can often be reached by increasing the tibial slope. This technique is implemented by surgeons in addition to the release of posterior ligaments assuming that "tibial roll-back" [18] during flexion prevents effects on the extension gap. 
The medial and lateral tibiofemoral contact points can be used to measure the extent of roll-back. Wretenberg et al. [19] reported on the extent to which the tibiofemoral contact points of the medial and lateral compartments shift with increasing flexion. The actual extent of the roll-back mechanism in this scenario is likely overestimated.

The goal of the current study is to assess the magnitude of the extension and flexion gaps as tibial slope increases, considering physiological movements and using the tibiofemoral contact points for measurements. The main hypothesis is that increasing tibial slope also enlarges the bony part of the extension gap.

In comparison to conventional methods of measuring extension and flexion gaps, i.e. under distraction, this analysis considers only the bony portion of the gaps without distraction, similar to the measured resection technique. This gap is defined as the "osseous resection gap." The starting measure is the exact amount of bone that needs to be resected from the femur and tibia in order to implant the prosthetic components (Fig. 1).

\section{Materials and methods}

After approval by the Ethics Commission Beider Basel (EKBB) of Switzerland, 20 (ten left and ten right) formalinfixed knee joint specimens from a university-level anatomy course were investigated. The average age of the donors was 81.9 years $( \pm 12.4)$, the mean body weight was $67.4 \mathrm{~kg}( \pm$ $14.8)$, and the mean body height was $164.8 \mathrm{~cm}( \pm 10.2)$. Ten specimens were from male and ten from female donors.

In order to get a specimen with intact capsule and ligaments, the femur, tibia, and fibula were each dissected $150 \mathrm{~mm}$ proximally or by two $6-\mathrm{mm}$ rods mounted in two drill holes that were $3 \mathrm{~cm}$ apart from each other in the coronal plane parallel to the knee joint axis. Skin, subcutaneous fat tissue, and muscle were predominantly resected.

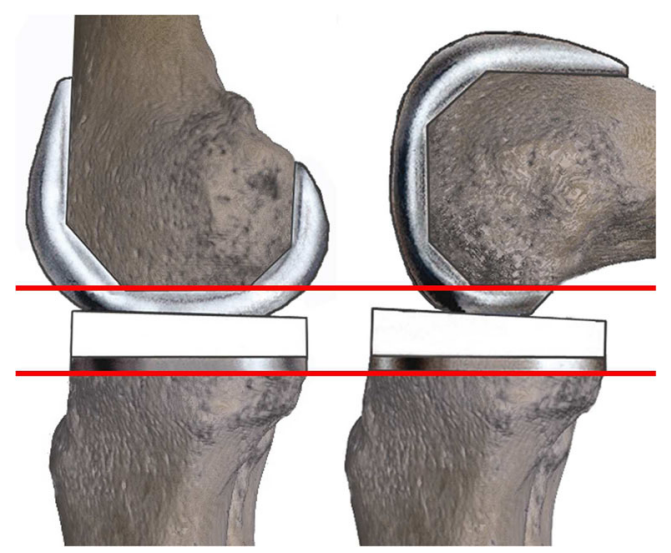

Fig. 1 Osseous resection gaps. The red lines designate cuts necessary for implantation of prosthetic components
A customized frame was constructed to fix the specimen during CT scan. It was essentially made out of plastic and carbon to allow artefact-free data acquisition (Fig. 2).

For attachment to the frame, the femur was enclosed in a plastic sleeve, and a carbon rod was fixed through the 6-mm canal. The tibia was rigidly fixed to the frame by two $6-\mathrm{mm}$ rods mounted in two drill holes that were $3 \mathrm{~cm}$ apart from each other in the coronal plane parallel to the knee joint axis.

Imaging was performed with a helical GE Lightspeed 16 row CT scanner (General Electric Healthcare Corporation, Waukesha, WI): $120 \mathrm{kV}$, slice thickness $0.625 \mathrm{~mm}$, Voxel depth $0.5 \mathrm{~mm}$, Voxel height $0.296875 \mathrm{~mm}$, and Voxel width $0.296875 \mathrm{~mm}$.

Two CT scans were performed per specimen, one in full extension and one at $90^{\circ}$ flexion.

Adjustment of data using a standardized coordinate system

The DICOM data was analyzed using the visualization software VGStudio Max 2.1.1 (Volume Graphics GmbH, Heidelberg, Germany), which allows high-precision measurements by means of a CT-based coordinate measurement technology [20]. The data from the knee specimens were imported into a defined coordinate system. The coordinate system was based on the reports of Grood et al. [21] as well as McPherson et al. [22]. The coordinate system was initially defined in full extension, since tibiofemoral rotation is least in this position [18, 22]. Two-dimensional reconstructions of the data sets in the sagittal, frontal, and transverse planes as well as a threedimensional reconstruction of the entire data volume per knee and axis were selected for monitor display. The transverse flexion-axis was determined by measuring movements of the

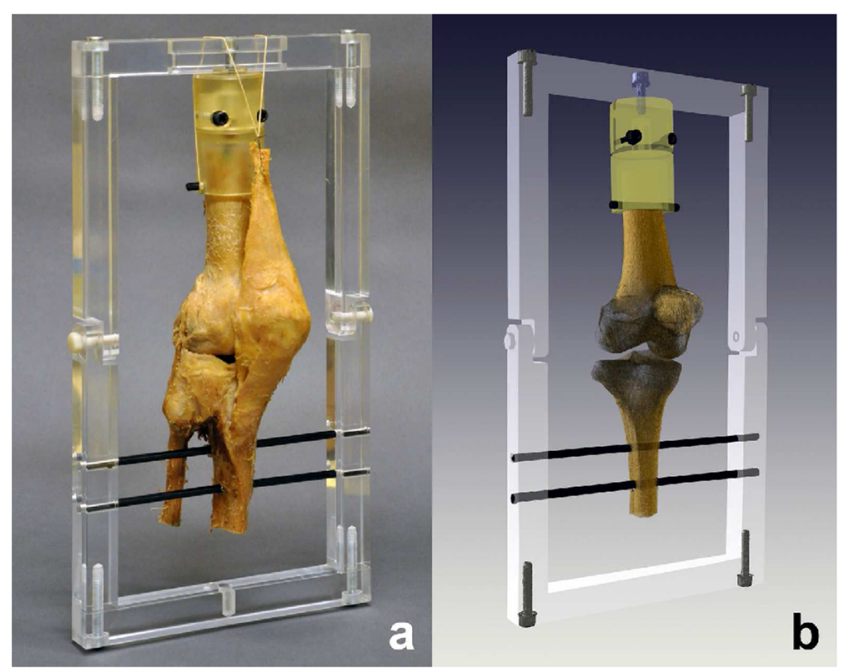

Fig. 2 Customized frame for CT scan. a) The frame was used for readings and the accurate positioning (motion) of the specimens. Although the frame does give the possibility to restrict the rotation of the femur, we did not use this option, in order to obtain a physiological movement. b) 3D CT reconstruction of the frame and specimen after CT scan 
flexion facet center (FFC) on the posterior femoral condyle. It runs nearly parallel to the joint axis [23, 24].

In the sagittal plane, tibial reference points (tRP) were determined [22]. The tRP is the intersection between the three spatial axes at the most distal edge of the posterior tibia, defined at the level of the center of the proximal tibial drill canal. The FFC and tRP span the frontal plane. The coordinate system could be established from the frontal plane (primary reference), the axis through the FFC (secondary reference), and the tRP as the origin (tertiary reference). After arrangement of the data with the knee in extension, the corresponding data in flexion was aligned with exact stacked projection using a software-integrated function (Fig. 3a).

Determination of the tibiofemoral contact points

We determined the tibiofemoral contact points (tfCPs) of the medial and lateral joint compartments in full extension and with $90^{\circ}$ of flexion according to the recommendations of Wretenberg et al. [19]. Because the tibiofemoral contact surfaces have a concave shape and thus, the tfCP is not located in the exact center of the contact surface, we selected the most inferior point of the medial and femoral condyles in the transverse plane, and included these in the calculation of the tfCP.

For each knee specimen, four tfCPs were determined, and for each of these, the distance to the transverse axis was measured. One each on the medial and lateral sides was measured in full extension, and again one each side in $90^{\circ}$ of flexion (Fig. 3b).

Virtual bone resection and measurement

Virtual bone resections were performed according to the descriptions of Cheng et al. [25]. Resection of the tibia was carried out $6 \mathrm{~mm}$ distal to the deepest point of the medial tibial plateau parallel to the "horizontal plane" [25]. This tibial cut was defined as the $0^{\circ}$ slope plane according to the standardized coordinate system. The distal femoral cut was made parallel to the tibial
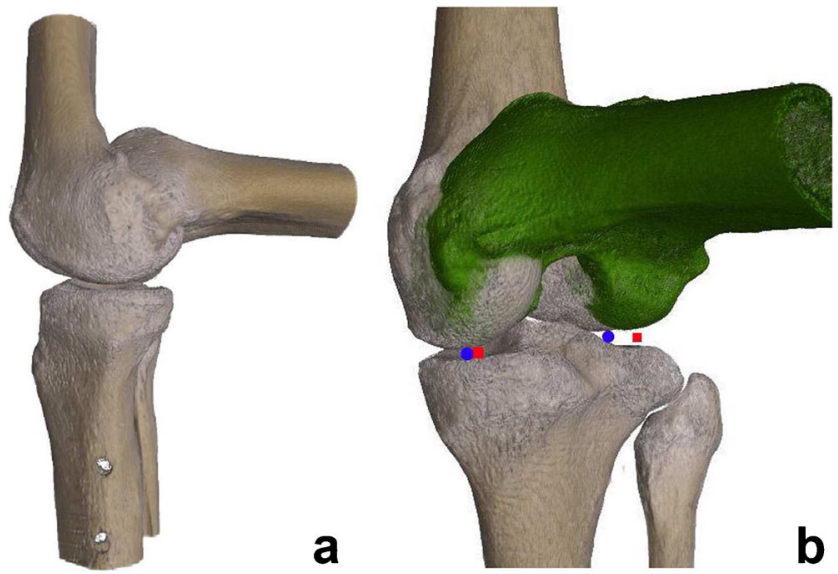

Fig. 3 Fusion of scans with specimen in extension and flexion. a) Exact stacking projection using the tibia to depict motion. b) Tibiofemoral contact point in extension (blue circle) and flexion (red square) in the medial (left) and lateral (right) compartments resection in full extension. Resection height was 9-mm proximal to the most distal point of the medial femoral condyle [25].

For the adjustment of the tibial slope, a pivot point was defined at the anterior edge of the tibial plateau. The longest mediolateral span of the tibial plateau was determined as the linear route. The route was halved, and the pivot point was set perpendicular to the midpoint along the anterior tibial border. The $0^{\circ}$ slope plane of the tibial plateau was inclined towards distal posterior from this pivot point to $3.5^{\circ}, 7^{\circ}$, and $10^{\circ}$ to perform the further virtual cuts (Fig. 4).

Using a distance measuring gauge (caliper), the separation between the distal femoral cut and the tibial resection plane could be measured in extension and in flexion with progressing slopes of $0^{\circ}, 3.5^{\circ}, 7^{\circ}$, and $10^{\circ}$ through the associated tfCPs. Sixteen measurements were produced per knee.

\section{Statistical methods}

A linear regression analysis was performed assuming the investigated variables were independent, i.e. the measurement of the distance between the femoral and tibial cuts through the corresponding tfCPs (in mm), motion (flexion vs. extension), and position (medial vs. lateral compartment).

We used a general estimating equation (GEE) regression model to obtain the desired independence of the respective measured variables in the identical knee. For each knee, a coefficient estimation was carried out using the Wald test [26]. Thus, we were able to ascertain whether the corresponding coefficient differed significantly from that with a $0^{\circ}$ slope or not.

In an additional GEE model, the gap distance, i.e. the investigated or independent variable, was correlated to the dependent

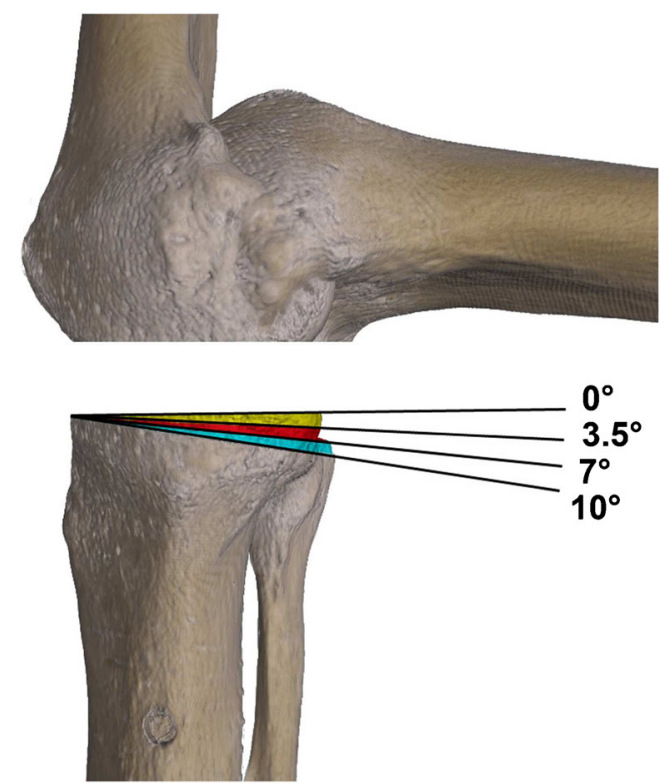

Fig. 4 Depiction of virtual osseus resection with tibial slopes of $0^{\circ}, 3.5^{\circ}$, $7^{\circ}$ and $10^{\circ}$ 
single explanatory categorical variable reflected by all 16 groups to attain the resulting estimation of the standard deviation.

All calculations were performed with R (R Development Core Team 2011, version 2.13.0).

\section{Results}

All tibiofemoral contact points (tfCPs) in the medial and lateral compartments shifted posteriorly, inferiorly, and laterally during flexion. The extent of movement between the contact points of the medial and lateral compartments varied. Overall, the posterior shift of the lateral tfCPs was greater than that of the medial tfCPs. In the medial compartment, the tfCPs shifted an average $4.4( \pm 2.2) \mathrm{mm}$ when flexing the knee from full extension to $90^{\circ}$, whereas the posterior shift of the lateral tfCPs averaged $16.3( \pm 5.0) \mathrm{mm}$.

The osseous resection gaps produced through the virtual osteotomy were equal for all knee specimens medially and laterally averaged $19.3( \pm 2.9 \mathrm{~mm})$ with a tibial slope of $0^{\circ}$.

Overall, 320 readings were produced. Per knee, two readings per position, compartment, and slope angle were performed, i.e. in total 16 readings per knee specimen.

The averaged gap increases per slope angle are summarized in Table 1.

In this area, the osseous resection gaps increased almost linearly with increasing slope in each position and at each measuring point. The relative measured gap increases by $10 \%$ from slope $0^{\circ}$ to $3.5^{\circ}$, from $3.5^{\circ}$ to $7^{\circ}$ and from $7^{\circ}$ to $10^{\circ}$ in both the medial and the lateral compartment. Again, the lateral compartment opened about $30 \%$ more in flexion than the other readings in each range (Fig. 5).

Calculation of average gap change according to slope increase in $1^{\circ}$ increments indicated a significant prosthetic gap change of $0.6 \mathrm{~mm}(p<0.001)$. Increasing the tibial slope did widen the lateral compartment significantly more than the medial one in $90^{\circ}$ of flexion $(p<0.001)$. For each $1^{\circ}$ increase of tibial slope, the osseous resection extension gaps increased $0.5( \pm 0.0) \mathrm{mm}$ medially, and $0.6( \pm 0.0) \mathrm{mm}$ laterally. In flexion, the medial osseous resection gap also averaged $0.6( \pm$ $0.1) \mathrm{mm}$, but laterally it was $0.9( \pm 0.1) \mathrm{mm}$ (Fig. 6).

\section{Discussion}

The aim of this study was to assess the influence of tibial resection on the osseous resection gaps in full extension and $90^{\circ}$ flexion according to slope angle and joint compartment.

All bone cuts were carried out virtually in 3D CT scans, after the data from knee joint specimens were adapted using a standardized coordinate system. At $0^{\circ}$ slope, the tibial cuts consisted only of the amount of bone necessary to allow prosthetic components to fill the space under load [27]. These gaps are differentiated from the typical extension and flexion gaps, which are defined under distraction of the knee joint $[6$, 7]. In the starting position (here at $0^{\circ}$ tibial slope), all four gaps, i.e. medial and lateral each in flexion and extension, were equally sized due to the virtual resection. The mean gap size of all 20 specimens averaged $19.3 \mathrm{~mm}$, with the accompanying standard deviation of $( \pm 2.9 \mathrm{~mm})$ reflecting basically the varying cartilage thicknesses present. This can be explained because of the resection technique as described by Tigani et al. [27], in which bony reference points were used. A total knee prosthesis that is symmetric in the area of the contact points would thus exactly fill in this osseous resection gap, and distraction of the knee in this state would, neglecting changes in the capsule/ligaments caused by the resection itself, lead to the typical extension and flexion gaps. It can be expressed by the following simplified relationship:

Extension or flexion gap $=$ elasticity of the soft tissue mantle + osseousresection gap

In cases of an "ideal" soft tissue mantle and optimal alignment of the components, adjustment of the leg axis, etc., the endoprosthesis perfectly fills the osseous resection gap in the area of the tibiofemoral contact points (tfCPs) during the complete range of flexion and extension. Thus, for this idealized case, the following can be assumed:

Osseous resection gap $_{\text {ideal }}=$ prosthetic gap

It is important to note that the prosthetic gap, according to the thickness of the prosthetic components, is thus always the
Table 1 Mean osseous resection gaps for motion (flexion vs. extension), and position (medial vs. lateral compartment) according to tibial slope

\begin{tabular}{llll}
\hline Slope $\left[{ }^{\circ}\right]$ & Motion & Medial mean gap $[\mathrm{mm}]$ & Lateral mean gap [mm] \\
\hline 0.0 & Full extension & $19.3(\mathrm{SD} 0.66)$ & 19.3 (SD 0.66) \\
3.5 & Full extension & $21.1(\mathrm{SD} 0.66)$ & 21.3 (SD 0.67) \\
7.0 & Full extension & $22.9(\mathrm{SD} 0.67)$ & $23.3(\mathrm{SD} 0.69)$ \\
10.0 & Full extension & $24.5(\mathrm{SD} 0.67)$ & 25.1 (SD 0.71) \\
0.0 & $90^{\circ}$ flexion & $19.3(\mathrm{SD} 0.66)$ & $19.3(\mathrm{SD} 0.66)$ \\
3.5 & $90^{\circ}$ flexion & $21.4(\mathrm{SD} 0.66)$ & $22.4(\mathrm{SD} 0.68)$ \\
7.0 & $90^{\circ}$ flexion & $23.4(\mathrm{SD} 0.65)$ & $25.3(\mathrm{SD} 0.70)$ \\
10.0 & $90^{\circ}$ flexion & $25.2(\mathrm{SD} 0.65)$ & $27.9(\mathrm{SD} 0.73)$ \\
\hline
\end{tabular}


Fig. 5 Mean of osseous resection gaps for position and motion according to increasing tibial slope
Changes of Extension and Flexion Gap as a Function of Slope

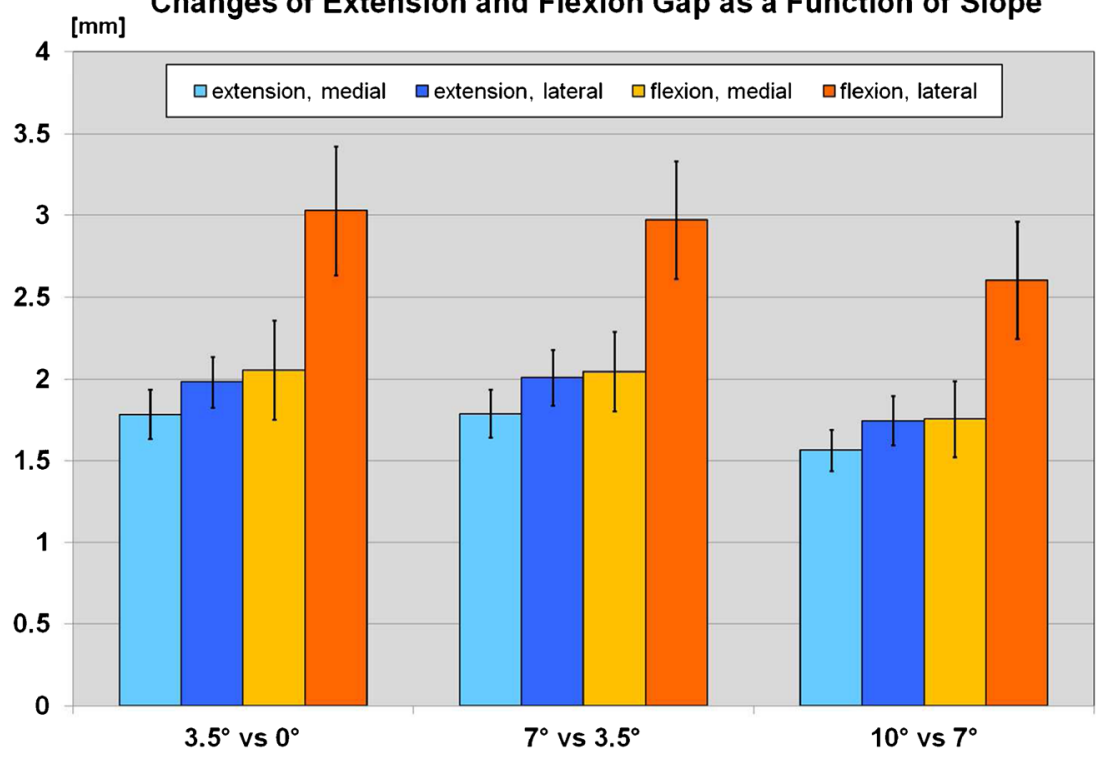

same size under loading in the $\mathrm{tfCP}$ regions. If additional bone is resected from one area, the osseous resection gap collapses under load to the size of the prosthetic gap, with concomitant relaxation of the soft tissue mantle. Conversely, too little bone resection will overstretch the soft tissue mantle.

The main limitations of this study result from the fact that formalin-fixed knee joint specimens have been used. Additionally the frame could have influenced the motion, leading to a non-physiologically restricted guidance. Therefore, we focused on the kinematics of the specimens during motion.

Positional changes of the tibiofemoral contact points (tfCPs) are correspondingly very important for physiological movement. The tfCPs can be used to measure the extent of roll-back, i.e. the anteroposterior shift of the contact surfaces between the femur and the tibia that occurs with increasing knee flexion. In this study, the
tfCP acted as a reference point to measure the distance between the femoral and tibial resection planes in each position and with each form of movement. In our cadaver study, similar to previous studies [3, 24], the tfCPs shifted only minimally posteriorly $(4.4 \pm$ $2.2 \mathrm{~mm}$ ) in the medial compartment from extension to flexion in $90^{\circ}$. However, the posterior shift in the lateral compartment was significantly larger $(16.3 \pm 5.0 \mathrm{~mm})$.

These values are in the range of data from previous studies, as collected by Freeman and Pinskerova [18] in an extensive review of the literature. As shown before our knee specimens were almost moving physiologically, despite formalin fixation and restricted guidance. Even more so, only the bony part of the gaps have been looked at in this study. This of course does not correspond with the complex intra-operative procedure with distraction force.
Fig. 6 Mean of osseous resection gaps per increasing $1^{\circ}$ increment of tibial slope

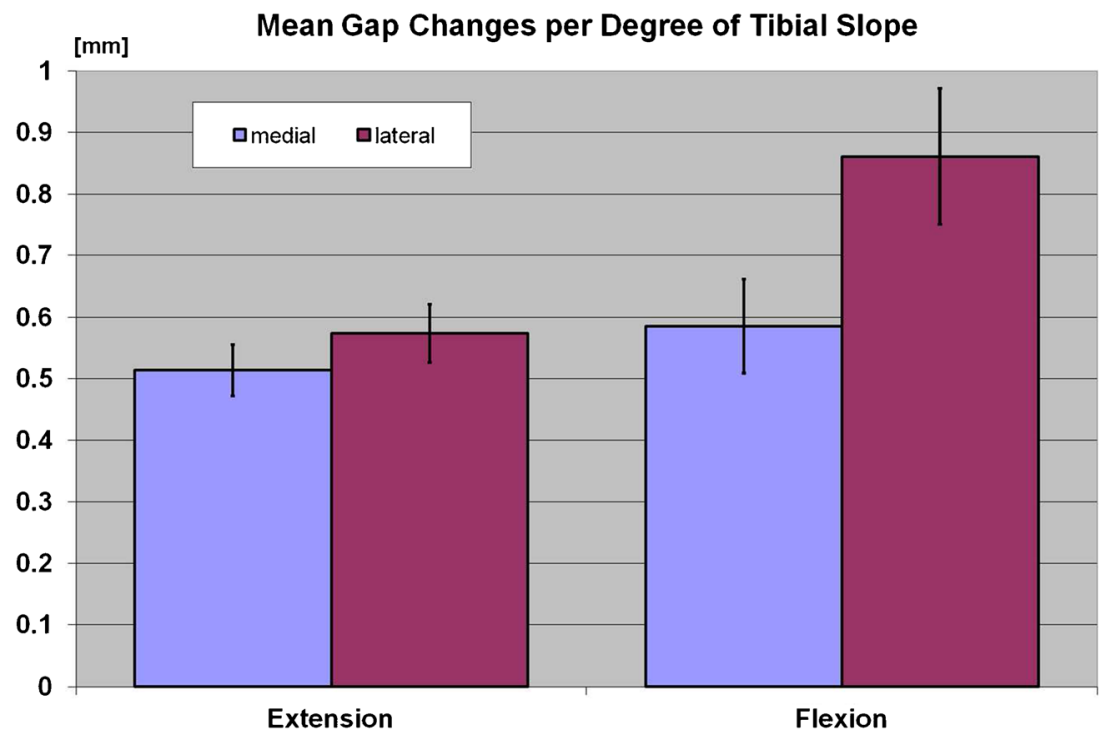


However, the bony part is of great importance for the postoperative alignment with weightbearing, and it is predictable by geometrical rules.

For each $1^{\circ}$ of increased tibial slope, the osseous resection gap increased approximately $0.5-0.6 \mathrm{~mm}$ in extension both medially and laterally and in flexion medially. In contrast, the lateral osseous resection gap increased by about $0.9 \mathrm{~mm}$ in flexion.

This larger effect on the lateral osseous resection gap is due to the larger lateral femoral roll-back, as occurs with physiological motion. When controlling the total knee jointmovement, however, often non-physiological motion and even paradoxical movement is documented $[28,29]$.

In such cases, the effects on the osseous resection gaps in extension would be even larger in comparison, although in the overall context here many factors must be considered that influence the sequence of motion after TKA. The intraoperatively observable effect of releasing the flexion gap by increasing tibial slope is explained by the corresponding release of the posterior ligament structures, as reported by Lombardi et al. [17] und Krakow [30].

\section{Conclusion}

The results of this study confirm that increasing the tibial slope affects not only the osseous resection gap in flexion, but also the osseous resection gap in extension $(p<0.001)$. Thus, we do not recommend equilibrating a narrow flexion gap by increasing the tibial slope in cases where the extension gap and the tibial slope are both appropriately adjusted with respect to leg-axis and tibial slope as planned. Instead, equilibration should be achieved via a ligament balancing procedure, without the use of additional bony resections.

Acknowledgments We wish to thank the collaborators of the Clinical Trial Unit CTU, University Hospital Basel, Switzerland: Thomas Zumbrunn, $\mathrm{PhD}$ and Thomas Fabbro, $\mathrm{PhD}$, for revising our methods and statistical analysis.

Conflict of interest The authors declare that they have no conflict of interest.

Open Access This article is distributed under the terms of the Creative Commons Attribution License which permits any use, distribution, and reproduction in any medium, provided the original author(s) and the source are credited.

\section{References}

1. Whiteside LA (1999) Selective ligament release in total knee arthroplasty of the knee in valgus. Clin Orthop Relat Res 367: $130-140$
2. Griffin FM, Insall JN, Scuderi GR (2000) Accuracy of soft tissue balancing in total knee arthroplasty. J Arthroplasty 15(8):970-973

3. Kadoya Y, Kobayashi A, Komatsu T, Nakagawa S, Yamano Y (2001) Effects of posterior cruciate ligament resection on the tibiofemoral joint gap. Clin Orthop Relat Res 391:210-217

4. Mihalko WM, Krackow KA (2006) Flexion and extension gap balancing in revision total knee arthroplasty. Clin Orthop Relat Res 446:121-126

5. Minoda Y, Sakawa A, Aihara M, Tada K, Kadoya Y, Kobayashi A (2007) Flexion gap preparation opens the extension gap in posterior cruciate ligament-retaining TKA. Knee Surg Sports Traumatol Arthrosc 15(11):1321-1325

6. Krackow KA, Mihalko WM (2001) The effects of severe femoral bone loss on the flexion extension joint space in revision total knee arthroplasty: a cadaveric analysis and clinical consequences. Orthopedics 24(2):121-126

7. Mihalko WM, Whiteside LA, Krackow KA (2003) Comparison of ligament-balancing techniques during total knee arthroplasty. J Bone Joint Surg Am 85-A(Suppl 4):132-135

8. Matsueda M, Gengerke TR, Murphy M, Lew WD, Gustilo RB (1999) Soft tissue release in total knee arthroplasty. Cadaver study using knees without deformities. Clin Orthop Relat Res 366:264-273

9. Heesterbeek PJ, Keijsers NL, Wymenga AB (2010) Ligament releases do not lead to increased postoperative varus-valgus laxity in flexion and extension: a prospective clinical study in 49 TKR patients. Knee Surg Sports Traumatol Arthrosc 18(2):187-93

10. Kanamiya T, Whiteside LA, Nakamura T, Mihalko WM, Steiger J, Naito M (2002) Ranawat Award paper. Effect of selective lateral ligament release on stability in knee arthroplasty. Clin Orthop Relat Res 404:24-31

11. Whiteside LA, Saeki K, Mihalko WM (2000) Functional medical ligament balancing in total knee arthroplasty. Clin Orthop Relat Res 380:45-57

12. Matsumoto T, Muratsu H, Tsumura N, Mizuno K, Kurosaka M, Kuroda R (2009) Soft tissue balance measurement in posteriorstabilized total knee arthroplasty with a navigation system. J Arthroplasty 24(3):358-364

13. Crottet D, Kowal J, Sarfert SA, Maeder T, Bleuler H, Nolte LP, Durselen L (2007) Ligament balancing in TKA: evaluation of a force-sensing device and the influence of patellar eversion and ligament release. J Biomech 40(8):1709-1715. doi:10.1016/j.jbiomech. 2006.08.004

14. Nowakowski AM, Majewski M, Muller-Gerbl M, Valderrabano V (2012) Measurement of knee joint gaps without bone resection: "physiologic" extension and flexion gaps in total knee arthroplasty are asymmetric and unequal and anterior and posterior cruciate ligament resections produce different gap changes. J Orthop Res 30(4):522-527

15. Nowakowski AM, Majewski M, Muller-Gerbl M, Valderrabano V (2011) Development of a force-determining tensor to measure "physiologic knee ligament gaps" without bone resection using a total knee arthroplasty approach. J Orthop Sci 16(1):56-63. doi:10.1007/ s00776-010-0015-1

16. Bellemans J, Robijns F, Duerinckx J, Banks S, Vandenneucker H (2005) The influence of tibial slope on maximal flexion after total knee arthroplasty. Knee Surg Sports Traumatol Arthrosc 13(3):193196. doi:10.1007/s00167-004-0557-x

17. Lombardi AV Jr, Berend KR, Aziz-Jacobo J, Davis MB (2008) Balancing the flexion gap: relationship between tibial slope and posterior cruciate ligament release and correlation with range of motion. J Bone Joint Surg Am 90(Suppl 4):121-132. doi:10.2106/ JBJS.H.00685

18. Freeman MA, Pinskerova V (2005) The movement of the normal tibio-femoral joint. J Biomech 38(2):197-208. doi:10.1016/j. jbiomech.2004.02.006 
19. Wretenberg P, Ramsey DK, Nemeth G (2002) Tibiofemoral contact points relative to flexion angle measured with MRI. Clin Biomech (Bristol, Avon) 17(6):477-485

20. Nowakowski AM, Muller-Gerbl M, Valderrabano V (2012) Assessment of knee implant alignment using coordinate measurement on three-dimensional computed tomography reconstructions. Surg Innov 19(4):375-384. doi:10.1177/1553350611429689

21. Grood ES, Suntay WJ (1983) A joint coordinate system for the clinical description of three-dimensional motions: application to the knee. J Biomech Eng 105(2):136-144

22. McPherson A, Karrholm J, Pinskerova V, Sosna A, Martelli S (2005) Imaging knee position using MRI, RSA/CT and 3D digitisation. $\mathrm{J}$ Biomech 38(2):263-268. doi:10.1016/j.jbiomech.2004.02.007

23. Iwaki H, Pinskerova V, Freeman MA (2000) Tibiofemoral movement 1: the shapes and relative movements of the femur and tibia in the unloaded cadaver knee. J Bone Joint Surg (Br) 82(8):1189-1195

24. Pinskerova V, Iwaki H, Freeman MA (2000) The shapes and relative movements of the femur and tibia at the knee. Orthopade 29(Suppl 1):S3-S5
25. Cheng FB, Ji XF, Lai Y, Feng JC, Zheng WX, Sun YF, Fu YW, Li YQ (2009) Three dimensional morphometry of the knee to design the total knee arthroplasty for Chinese population. Knee 16(5):341-347. doi:10.1016/j.knee.2008.12.019

26. Wald A (1943) On a statistical generalization of metric spaces. Proc Natl Acad Sci USA 29(6):196-197

27. Tigani D, Sabbioni G, Ben Ayad R, Filanti M, Rani N, Del Piccolo N (2010) Comparison between two computer-assisted total knee arthroplasty: gap-balancing versus measured resection technique. Knee Surg Sports Traumatol Arthrosc 18(10):1304-1310. doi:10. 1007/s00167-010-1124-2

28. Stiehl JB, Dennis DA, Komistek RD, Keblish PA (1997) In vivo kinematic analysis of a mobile bearing total knee prosthesis. Clin Orthop Relat Res 345:60-66

29. Dennis DA, Komistek RD, Colwell CE Jr, Ranawat CS, Scott RD, Thornhill TS, Lapp MA (1998) In vivo anteroposterior femorotibial translation of total knee arthroplasty: a multicenter analysis. Clin Orthop Relat Res 356:47-57

30. Krackow KA (1990) The technique of total knee arthroplasty. C.V. Mosby, St Louis 